pathways that upregulate GSL synthesis. Because different bacteria and bacterial products activated GSLspecific T cells, it is probable that several different pattern-recognition receptors and signalling pathways converge on the GSL biosynthesis machinery. Notably, only intact LPS could prime the APCs for optimal T-cell activation; lipid A or modified LPS was ineffective in this respect.

With regard to the physiological functions of GSL-specific T cells, these remain contentious, but because these cells respond to APCs that are primed by microbial infection, it is probable that they have a role in either promoting or modulating the initial immune response to infection. After resolution of infection, these self-GSL-specific $\mathrm{T}$ cells are a repository of potentially autoreactive lymphocytes.

Shannon Amoils, Assistant editor, Nature Reviews Microbiology

(2) References and links ORIGINAL RESEARCH PAPER De Libero, G. et al. Bacterial infections promote $T$ cell recognition of self-glycolipids. Immunity 22, 763-772 (2005) FURTHER READING Kronenberg, M. and Kinjo, Y. Infection, autoimmunity, and glycolipids: $T$ cells detect microbes through self-recognition. Immunity 22, 657-659 (2005)

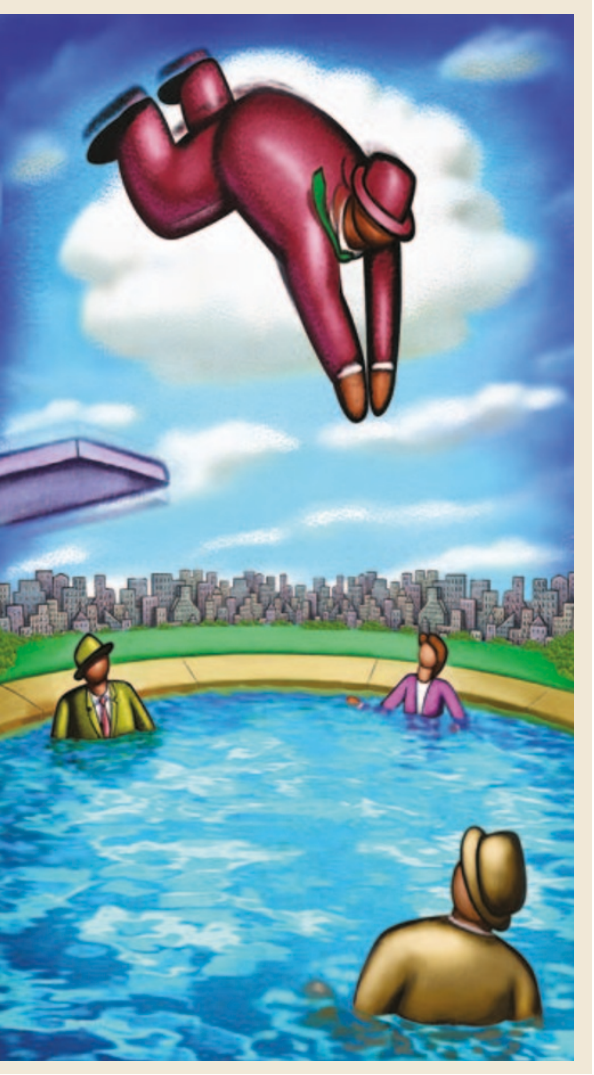

LYMPHOCYTE RESPONSES

\section{How RAG2 cycles}

The generation of lymphocyte-specific antigen receptors by $\mathrm{V}(\mathrm{D}) \mathrm{J}$ recombination is controlled by levels of recombination-activating gene 2

(RAG2) protein, which accumulates during the G1 (gap 1) phase of the cell cycle but is rapidly degraded at the G1-S (synthesis)-phase transition, continuing until the next entry to G1. This ensures that the double-stranded DNA breaks that are created by RAG 2 can be repaired correctly by non-homologous end joining (NHEJ), which is also most active during G1, and this might prevent genomic instability. A recent study in Molecular Cell has determined the molecular mechanisms that link RAG2 degradation to the cell cycle.

The authors used a cell-free in vitro system consisting of an extract of HeLa cells synchronized in $S$ phase to which RAG 2 constructs - in the form of a glutathione $S$-transferase fusion protein containing the carboxy-terminal 89 amino-acid residues of RAG2 (denoted GST-R2CT89) - were added. To confirm that this system recapitulates the in vivo situation, they showed that a mutation of the threonine residue at position 490 of RAG2 that inhibits degradation in vivo also reduced the degradation of RAG2 (in the form of GSTR2CT89(Thr490Ala)) in the in vitro system.

In the cell-free S-phase system, addition of a proteasome inhibitor or ubiquitin mutants that inhibit ubiquitin branching impaired GST-R2CT89 degradation, and the involvement of the ubiquitin-proteasome pathway in RAG2 degradation was then confirmed in S-phase cells. By contrast, the levels of GST-R2CT89(Thr490Ala) were not affected by proteasome inhibition. Previous genetic evidence has indicated that the cyclin-dependent kinase (CDK) inhibitor $\mathrm{p} 21$ and the cyclin-A-CDK2 complex are negative and positive regulators, respectively, of this RAG2-degradation pathway. Indeed, in the cell-free system, addition of p21 to, or depletion of CDK2 or cyclin A from, the S-phase extract inhibited the ubiquitylation and degradation of GST-R2CT89.

The substrate selectivity of ubiquitylation is mainly determined by E3 ligases, so the authors next looked for an E3 ligase that recognizes the carboxyl terminus of RAG2 in fractions of S-phase extract. One fraction contained substantial ubiquitylation activity against GST-R2CT89, and this fraction was enriched for SKP2, which is a subunit of the SCF-family multicomponent E3 ligases. Depletion of SKP2 from the S-phase extract markedly reduced ubiquitylation and degradation of GST-R2CT89.

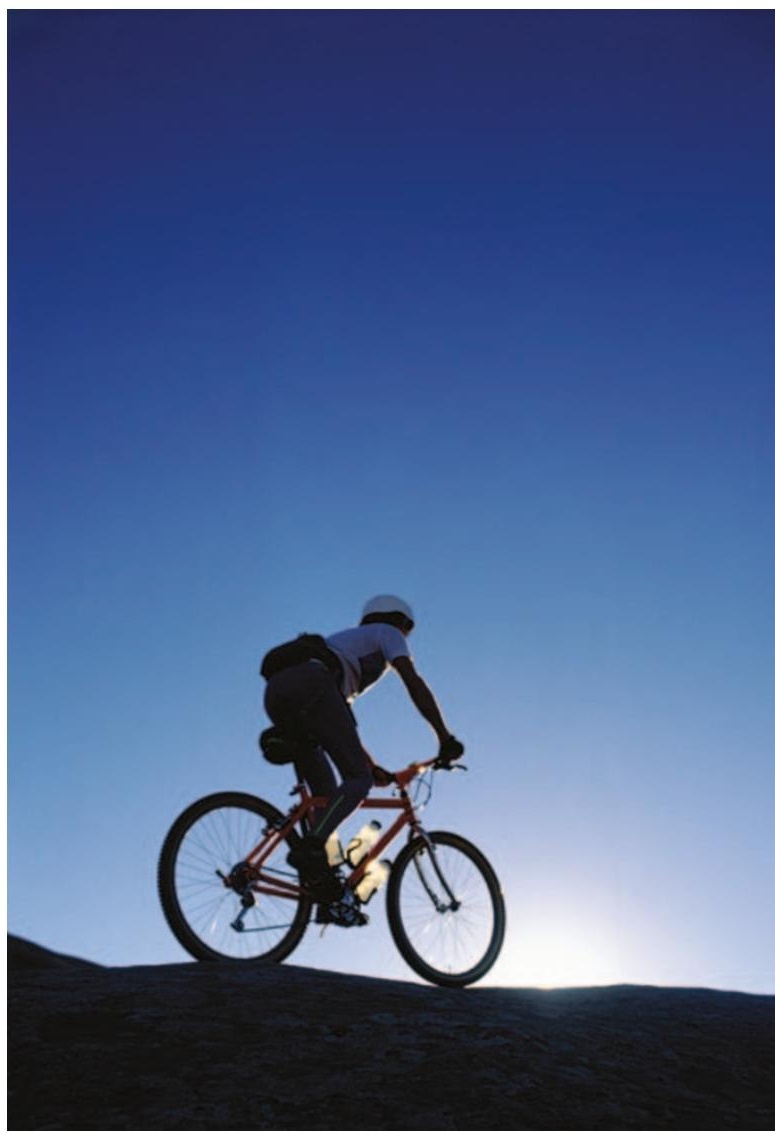

SKP2 was shown to interact specifically with the Thr490-phosphorylated form of RAG2 but not with other phosphorylated or unphosphorylated forms. The low-degradation activity of a G1-phase extract could be restored by addition of cyclin A, CDK2 and SKP2, showing that these are the limiting factors for RAG2 degradation. The authors suggest a model in which RAG2 is phosphorylated at Thr490 by cyclin-A-CDK2, which creates a binding site for SKP2, leading to ubiquitylation and degradation. The involvement of SKP2 in the destruction of RAG2 was confirmed in vivo: RAG2 accumulation was uncoupled from the cell cycle in thymocytes from a SKP2-deficient mouse, and expression of a dominant-negative mutant of SKP2 in HeLa cells led to increased accumulation of RAG2 protein.

SKP2 is a central regulator of cell-cycle progression, so the finding that SKP2 is also involved in RAG2 degradation provides a direct mechanistic link between DNA recombination and the cell cycle. This ensures that RAG2 expression is restricted to $\mathrm{G} 1$ to coordinate the generation of RAG-induced DNA breaks with repair by NHEJ.

Kirsty Minton

(2) References and links

ORIGINAL RESEARCH PAPER Jiang, H. et al. Ubiquitylation of RAG-2 by Skp2-SCF links destruction of the V(D)J recombinase to the cell cycle. Mol. Cell 18, 699-709 (2005) 